\section{DOES PHENOBARBITAL IMPROVE THE EFFECTIVENESS OF THERAPEUTIC HYPOTHERMIA IN INFANTS WITH HYPOXIC- ISCHEMIC ENCEPHALOPATHY?}

\author{
S. Sarkar ${ }^{1}$, I. Bhagat ${ }^{2}$, R.E. Dechert ${ }^{1}$, \\ R.E. Schumacher ${ }^{1}$, S.M. Donn ${ }^{1}$, J.D. Barks ${ }^{1}$ \\ ${ }^{1}$ Department of Pediatrics, Division of Neonatal- \\ Perinatal Medicine, University of Michigan Health \\ System, ${ }^{2}$ Pediatrics, St. Joseph Mercy Hospital, \\ Ann Arbor, MI, USA
}

Objective: To determine whether Phenobarbital(PB) given before therapeutic hypothermia to infants with hypoxic-ischemic encephalopathy (HIE) augments the neuroprotective efficacy of hypothermia.

Methods: Records of 68 asphyxiated infants of $\geq$ 36 weeks' gestation, who received hypothermia for moderate or severe HIE were reviewed. Some of these infants received PB prophylactically or for clinical seizures. All surviving infants had later brain MRI. The composite primary outcome of HIErelated neonatal death, and MRI abnormalities consistent with hypoxic-ischemic brain injury, were compared between the infants who received $\mathrm{PB}$ before initiation of hypothermia (PB group, $n=36$ ) and the infants who did not receive $\mathrm{PB}$ before or during hypothermia (no PB group, $n=32$ ). Logistic regression analysis determined which of the prehypothermia clinical and laboratory variables predict the primary outcome.

Results: The two groups were similar for severity of asphyxia as assessed by Apgar scores, initial blood $\mathrm{pH}$ and base deficit, early neurologic examination, and presence of an intrapartum sentinel event. The composite primary outcome was more frequent in infants from the PB group (PB 78\% versus No PB 44\%, $p=0.006$, OR 4.5, 95\% Cl 1.6 12.8). Multivariate analysis identified only the PB receipt before initiation of hypothermia $(p=0.002$, OR 9.5, $95 \% \mathrm{Cl} 2.3$ 39.5), and placental abruption to be independently associated with a worse primary outcome.

Conclusions: The combination of therapeutic hypothermia and PB may be associated with an increase in the composite outcome of HIE-related neonatal death and the brain MRI abnormalities, but the long term outcomes have not yet been evaluated.

\section{FETAL ALCOHOL SYNDROME (FAS); ARE WE GETTING THE MESSAGE ACROSS? AUSTRALIAN, DANISH AND BRITISH DATA ANALYSED AND COMPARED}

\author{
A.M. Winstone ${ }^{1}$, U. Kesmodel ${ }^{2,3}$, J. Payne ${ }^{4}$, \\ E.J. Elliott ${ }^{5,6}$ \\ ${ }^{1}$ Addenbrookes Hospital, Cambridge, UK, \\ ${ }^{2}$ School of Public Health, Department of \\ Epidemiology, Aarhus University, ${ }^{3}$ Department \\ of Obstetrics \& Gynaecology, Aarhus University \\ Hospital, Skejby, Aarhus, Denmark, ${ }^{4}$ Division of \\ Population Sciences, Telethon Institute for Child \\ Health Research, West Perth, WA, ${ }^{5}$ Professor \\ Paediatrics and Child Health, University of Sydney, \\ ${ }^{6}$ Department of Paediatrics, Children's Hospital at \\ Westmead, Sydney, NSW, Australia
}

Background and aims: To report on surveys of health professionals: 1443 in Western Australia, 165 in Denmark \& 40 in the UK who were asked about their knowledge \& practice regarding FAS \& alcohol use during pregnancy.

Methods: In Australia \& in the UK an anonymous postal questionnaire consisting of 44 multiple choice, Likert scale \& open-questions was used. The Danish study used face-to-face interviews (open-ended questions). Analysis: descriptive statistics \& the Statistical Package for the Social Sciences (SPSS).

Results: Study response rates; Australia 79\%, Denmark $77.5 \%$ \& UK $72.5 \%$.

$12 \%$ of Australian participants could identify the 4 FAS diagnostic features compared with $10 \%$ in the UK (no DK data).

In Australia (A) $45 \%$ routinely asked pregnant women about alcohol intake compared with $85 \%$ in Denmark (DK) \& $100 \%$ in the UK, but only $25 \%$ (A), 64\% (DK) \& 55\% (UK) routinely gave advice. Abstaining from binge-drinking ( $n=\geq 5$ drinks) was advised by $29 \%$ (A), $28 \%$ (DK) \& by none in the UK. Total alcohol abstention was recommended by $87 \%$ in Australia, $28 \%$ in Denmark \& $100 \%$ in the UK.

Only $13 \%$ of Australian \& none of the Danish or UK participants provided information in accordance with their countries' national guidelines.

Conclusions: These international data suggest that knowledge about FAS is limited, that consistent, evidence-based alcohol advice is not provided \& that 